\title{
Reliability test results of the interconnect structures of the front-end hybrids for the CMS Phase-2 Tracker Upgrade
}

\author{
Mark Istvan Kovacs ${ }^{1}$ \\ CERN \\ CH-1211 Geneva 23, Switzerland \\ E-mail:mark.istvan.kovacs@cern.ch

\section{B. Allongue, G. Blanchot, T. Gadek, R. Gajanec} \\ CERN \\ CH-1211 Geneva 23, Switzerland \\ Bruno-Andre.Allongue@cern.ch,georges.blanchot@cern.ch,tomasz.gadek@cern.ch
}

\section{F. Andre}

Télécom Physique Strasbourg

Université de Strasbourg, 300 Bd Sébastien Brant, 67400 Illkirch-Graffenstaden, France

florianandre.fr@gmail.com

Abstract

High Density Interconnect (HDI) hybrids are being developed for the CMS Tracker Phase Two Upgrade for the HL-LHC. These hybrids are carbon fibre reinforced flexible circuits with flip-chips, passives and connectors. Their operational lifetime is determined by the reliability of the solder joints of the surface mount components (flip-chips, passives, connectors) and the copper traces and vias in the hybrid substrate. Specific test coupons were exposed to accelerated thermal stress cycles, aiming to test the reliability of the solder joints, vias and traces. Results from different suppliers and technologies will be evaluated and compared.

\section{${ }^{1}$ Speaker}




\section{Introduction}

Components for the Compact Muon Solenoid (CMS) Tracker Phase-2 Upgrade for the High Luminosity Large Hadron Collider (HL-LHC) are currently under development. In order to meet the highly demanding material budget, bandwidth and resolution requirements of the upgrade [1], High Density Interconnect (HDI) flexible circuits are being developed. These circuits are reinforced with high thermal conductivity and tensile modulus carbon fibre composite laminates. The reinforced circuits are equipped with fine-pitch connectors, passive components and up to 9 flip-chip Application Specific Integrated Circuits (ASICs). The planned lifetime of the electronics is approximately 15 years in the tracker environment, but carbon-fibre reinforced HDI flexible circuits were never used in the CMS Tracker before. In order to estimate the reliability of these assemblies and to control their quality, test coupons were added to the production panels of many front-end hybrid circuit prototypes. The coupons contain different test geometries in order to test the different circuit elements that can fail during the lifetime of the object. These elements are vias, fine traces and the solder interconnection of the surface mount components. Different test coupons were assembled with Pb37Sn63 and SAC305 solder paste by several manufacturers. The coupons were exposed to thermal cycling test for approximately 1000 cycles and their resistance was monitored for the full test duration. The failures found were analysed to identify the failure modes. This paper will present the test coupon design, the test method, the test system design, the failures with their failure mode and the conclusion of the test followed by the future plans.

\section{Test coupons and test structures}

The test coupons are made with the same technology and build-up as the hybrids and they are located on the manufacturing panel. All test coupons are assembled on carbon-fibre laminate stiffeners in order to achieve the same build-up as in the hybrids. Each different type of test coupon can contain up to three different test structures. A via daisy-chain, a fine-line and a flipchip daisy-chain structure. All the three test structures are illustrated in Figure 1. The via daisychain structure uses copper filled microvias with $110 \mu \mathrm{m}$ diameter capture pad and 25 to $50 \mu \mathrm{m}$ drill diameter. The vias are going through all layer pairs in a staggered arrangement, similarly as it is implemented in the hybrid's design to model the hybrid's interconnections as good as possible.

The fine-line test structure consists of one or two $45 \mu \mathrm{m}$ traces routed next to each-other with 45 $\mu \mathrm{m}$ separation. The fine trace is up to $1.6 \mathrm{~m}$ long and in the case with two traces next to each other, it can sense shorts formed between the fine traces.

The flip-chip daisy-chain test structure consists of a via-in pad pattern similar to the one in the via daisy-chain, but in this case a test flip-chip is assembled on the coupon in order to test the reliability of the bump bond connections. The test flip-chip has 317 bumps with $254 \mu \mathrm{m}$ pitch and around $140 \mu \mathrm{m}$ width. Two types of bump compositions were used: Sn63Pb37 and SAC305. Two types of flip-chip surface mount pads were used: copper defined in one case and solder mask defined in all the other cases.

Not all the test coupons are designed with all the test structures. The test coupon \#1 is only equipped with a via daisy-chain interconnecting 6504 vias. This coupon was manufactured by one manufacturer and three instances were irradiated at 1 MGy gamma ray.

The test coupon \#2 is equipped with all types of the test structures. A via daisy-chain with 3564 vias staggered, 5 flip-chip daisy-chain and a fine-line test structure with $1.6 \mathrm{~m}$ of total length. These coupons are equipped with test chips equipped with SAC305 bumps. 
TWEPP-18 - Reliability test results of the interconnect structures of the front-end hybrids for the CMS Phase-2 Tracker Upgrade

Mark Istvan Kovacs

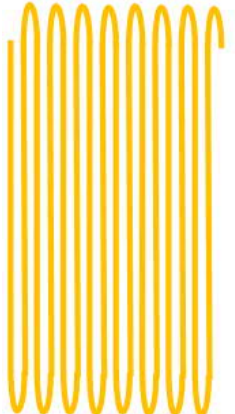

Fine line

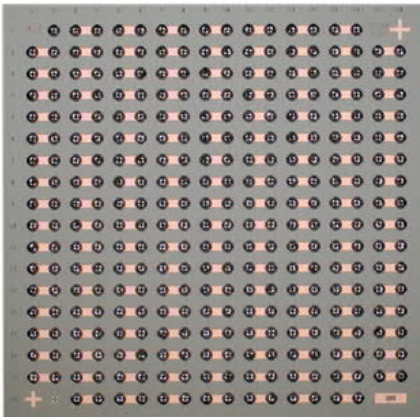

Daisy-chain flip-chip

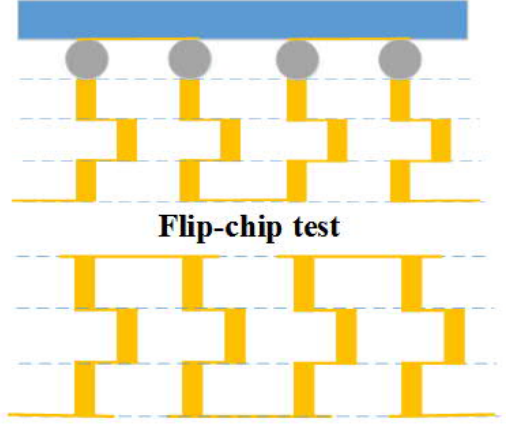

Via daisy-chain

Figure 1: Illustration of the test coupon test structures and the "active side" of the test flip-chip.

The test coupon \#3 is also equipped with all the test structures. It has a via daisy-chain with 1158 vias, one flip-chip daisy chain and a fine-line test structure with $1.4 \mathrm{~m}$ total length. Three different manufacturers produced these coupons. All of them are equipped with Sn63Pb37 bumped flip-chips. One coupon is produced with copper defined flip-chip surface mount pads.

The fourth type of test coupon was designed to test the reliability of the Panasonic A35S connector family. Ten of these coupons were irradiated at $1 \mathrm{MGy}$ gamma ray and five of these had five plug-unplug cycles. This is also true for the non-irradiated ones. The different types of test coupons are shown in Figure 2. The summary of test coupons is listed in Table 1.
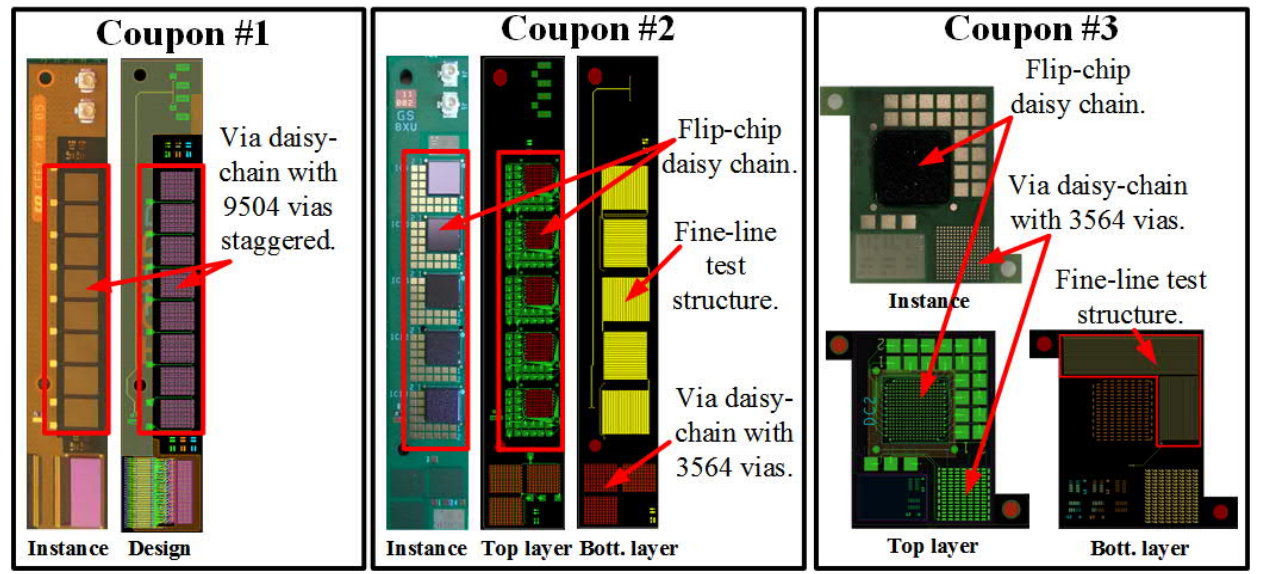

Figure 2: The instances and the design of different test coupons used during the tests.

\begin{tabular}{|c|c|c|c|c|c|}
\hline Coupon type & Flip-chip DC & Via DC & Fine line & Other information & Pieces \\
\hline Coupon \#1 & No & Yes & No & Irradiated & 3 \\
\hline Coupon \#1 & No & Yes & No & - & 4 \\
\hline Coupon \#2 & Yes & Yes & Yes & SAC305, SM defined & 17 \\
\hline Coupon \#2 & No & Yes & Yes & - & 11 \\
\hline Coupon \#3V & No & Yes & Yes & SnPb, SM defined & 11 \\
\hline Coupon \#3E & Yes & Yes & Yes & SnPb, Cu defined & 8 \\
\hline Coupon \#3E & No & Yes & Yes & SnPb, Cu defined & 6 \\
\hline Coupon \#3A & Yes & Yes & Yes & SnPb, SM defined & 14 \\
\hline Conn. coup. & - & - & - & Irradiated, 5 cycles & 5 \\
\hline Conn. coup. & - & - & - & Irradiated, no cycles & 4 \\
\hline Conn. coup. & - & - & - & 5 cycles & 5 \\
\hline Conn. coup. & - & - & - & no cycles & 5 \\
\hline
\end{tabular}

Table 1: Summary of test coupons tested during the tests. 


\section{Test method and test system}

The reliability testing is based on thermal cycling. The applied thermal cycle is running between $-35{ }^{\circ} \mathrm{C}$ to $65{ }^{\circ} \mathrm{C}$ with 15 minutes of dwell time and 15 minutes of temperature ramp. The maximum temperature change rate is $7{ }^{\circ} \mathrm{C} / \mathrm{min}$ to avoid thermal shock. Dry air injection was activated during the tests to prevent condensation on the samples. The cycles and the test system were programmed and designed based on the recommendations in IPC-SM-785.[2] The test system consists of two functional parts: a resistance measurement system and a transient event detection system. The transient detection system is detecting resistance transients above the threshold of $60 \Omega$ for at least $1 \mu$ s duration. This system is implemented with counters in Arduino Due microcontroller boards. The resistance measurement is implemented with Keithley 2000 multimeters and a Keithley 7001 switch. The system monitors the resistance of 80 channels every 5 minutes. A test interconnect board hosts a low dropout regulator to provide a reference voltage. This voltage is measured every 5 minutes. Reference resistors are connected in series with the test coupons and the coupons are connected to a common ground point. The system monitors the voltage drop on each coupon to calculate the resistance. The temperature of the climatic chamber is measured in each measurement point for all the channels. Figure 3 shows the schematic diagram of the system.

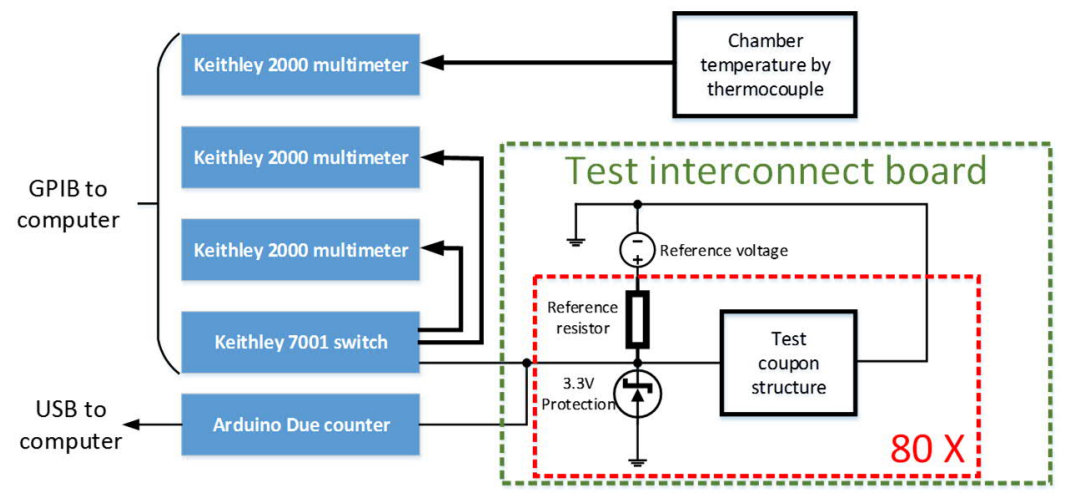

Figure 3: Schematic diagram of the reliability test system used for monitoring the coupons.

\subsection{Performance of the test system}

The test system is equipped with three reference resistors to monitor the performance of the system. These resistors are placed on the system interconnect board, which is placed outside of the climatic chamber. The resistors are measured in every measurement cycle. The system showed excellent stability, less than $0.1 \%$ deviation was observed from the first measured value during the test. In the case of the event detection system, events were triggered in most cases when a resistance increase was detected by the resistance measurement system. In some cases no events were detected by the event detection system, but significant resistance increase was registered by the resistance measurement system. In general, the resistance monitoring could indicate precisely when a failure occurred during the test.

\section{Test results}

The results were analysed for each test coupon and the failure modes were studied with cross section polishes, microscope analysis and SEM analysis. No failures have developed in the following test coupons: coupon \#1 via daisy-chain, coupon \#3V via daisy-chain, coupon \#3V fine-line, coupon \#2 fine-line, coupon \#3 A flip-chip daisy-chain and no failures occurred in the connector test coupons. Where failures were found, design, assembly or PCB manufacturing problems caused these failures. Figure 4 and Figure 5 illustrates the failure modes found in the test coupons. Some of the design and assembly failures were caused by the CTE mismatch of 
TWEPP-18 - Reliability test results of the interconnect structures of the front-end hybrids for the CMS Phase-2 Tracker Upgrade

Mark Istvan Kovacs

the flex circuit and the carbon-fibre stiffener. During the assembly, the surface of the flex circuit warps and the bump bonds cannot form properly. Design problem is found near the solder pads of the test coupons, the thin trace connecting to the pad can easily crack during the thermal cycles. On the \#3V test coupons the soldermask opening size was not tuned to the solder mask layer thickness and the flip-chip assembly failed due to the too small opening size. The summary of the failures and failure modes are listed in Table 2.

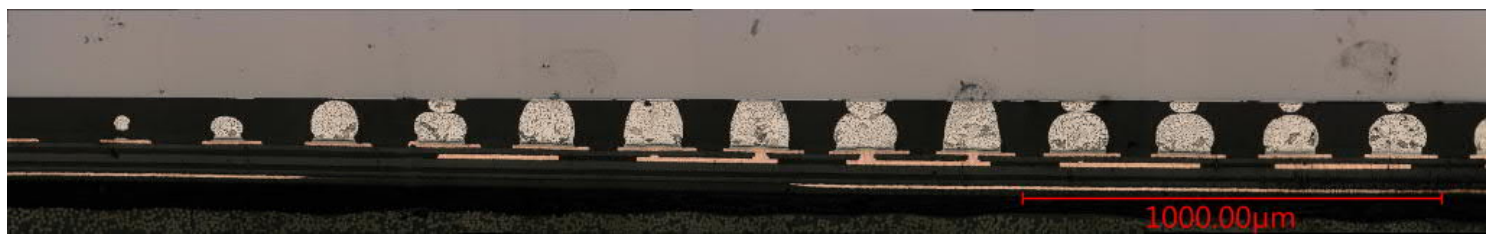

Figure 4: Bump bonds failed to form correctly due to the substrate and stiffener CTE mismatch.


Figure 5: Other failures caused by design and PCB manufacturing problems.

\begin{tabular}{|c|c|c|c|c|}
\hline Coupon type & $\begin{array}{c}\text { Solder pad fine } \\
\text { trace crack }\end{array}$ & $\begin{array}{c}\text { Too small } \\
\text { SM opening }\end{array}$ & $\begin{array}{c}\text { Warpage issue } \\
\text { during assembly }\end{array}$ & $\begin{array}{c}\text { Not yet } \\
\text { investigated }\end{array}$ \\
\hline Coupon \#2 via daisy-chain & 2 & & \\
\hline Coupon \#3A via daisy-chain & 2 & & \\
\hline Coupon \#3E via daisy-chain & 1 & & \\
\hline Coupon \#3A fine line & 2 & & \\
\hline Coupon \#3E fine line & 2 & & 11 & \\
\hline Coupon \#2 flip-chip daisy-chain & & 14 & \\
\hline Coupon \#3V flip-chip daisy-chain & & & \\
\hline Coupon \#3E flip-chip daisy-chain & & & \\
\hline
\end{tabular}

Table 2: Summary of the failures found and their failure mode.

\section{Conclusions and future work}

Different types of test coupons were thermal cycled for more than 1000 cycles. The test coupons developed different failure modes, but none of them were fatigue failures. The test was useful to find manufacturing and design issues. These issues are currently being solved and new design solutions are already implemented. Thermal cycling tests will continue in the future with the coupons which did not form failures and new coupons will be produced. The final goal is to find fatigue failures and estimate the expected lifetime of different assemblies in the Tracker environment. During the production phase of the Tracker front-end hybrids, test coupons will be produced and will be used to control the quality of the supplied batches.

\section{References}

[1] CMS collaboration. The Phase-2 Upgrade of the CMS Tracker Technical Design Report CMSTDR-17-001

[2] IPC-SM-785 Guidelines for Accelerated Reliability Testing of Surface Mount Solder Attachments 